\title{
Second Preimage Attack on 3-Pass HAVAL and Partial Key-Recovery Attacks on HMAC/NMAC-3-Pass HAVAL
}

\author{
Eunjin Lee ${ }^{1}$, Donghoon Chang ${ }^{1}$, Jongsung Kim ${ }^{1}$, Jaechul Sung ${ }^{2}$, \\ and Seokhie Hong ${ }^{1}$ \\ ${ }^{1}$ Center for Information Security Technologies(CIST), \\ Korea University, Seoul, Korea \\ \{walgadak, pointchang, joshep, hsh\}@cist.korea.ac.kr \\ ${ }^{2}$ University of Seoul,Seoul, Korea \\ jcsung@uos.ac.kr
}

\begin{abstract}
In 1992, Zheng, Pieprzyk and Seberry proposed a one-way hashing algorithm called HAVAL, which compresses a message of arbitrary length into a digest of 128, 160, 192, 224 or 256 bits. It operates in so called passes where each pass contains 32 steps. The number of passes can be chosen equal to 3,4 or 5 . In this paper, we devise a new differential path of 3-pass HAVAL with probability $2^{-114}$, which allows us to design a second preimage attack on 3-pass HAVAL and partial key recovery attacks on HMAC/NMAC-3-pass HAVAL. Our partial keyrecovery attack works with $2^{122}$ oracle queries, $5 \cdot 2^{32}$ memory bytes and $2^{96} 3$-pass HAVAL computations.
\end{abstract}

Keywords: HAVAL, NMAC, HMAC, Second preimage attack, Key recovery attack.

\section{Introduction}

In 2004 and 2005, Biham et al. and Wang et al. published several important cryptanalytic articles [12 12 13 1415] that demonstrate efficient collision search algorithms for the MD4-family of hash functions. Their proposed neutral-bit and message modification techniques make it possible to significantly improve previous known collision attacks on MD4, MD5, HAVAL, RIPEMD, SHA-0 and SHA-1 3910 17], including the second preimage attack on MD4 which finds a second preimage for a random message with probability $2^{-56}[18$.

There have also been several articles that present attacks on NMAC and HMAC based on the MD4 family. In 2006, Kim et al. first proposed distinguishing and forgery attacks on NMAC and HMAC based on the full or reduced HAVAL, MD4, MD5, SHA-0 and SHA-1 [7] and Contini and Yin presented forgery and partial key recovery attacks on HMAC/NMAC-MD4, -SHA-0, -reduced 34-round SHA-1 and NMAC-MD5 4. More recently, full key-recovery attacks on HMAC/NMACMD4, reduced 61-round SHA-1 and NMAC-MD5 were proposed in FC 2007 [8] and in CRYPTO 2007 [6]. 
The motivation of this paper is that 1) there are strong collision producing differentials of HAVAL for collision attacks [1011, but no differential of HAVAL has been proposed for second preimage attacks, and 2) there are distinguishing/forgery attacks on HMAC/NMAC-HAVAL [7, but no key-recovery attack has been proposed. This paper investigates if 3-pass HAVAL and HMAC/NMAC3-pass HAVAL are vulnerable to the second preimage and partial key recovery attacks, respectively. (After our submission, we learned that Hongbo Yu worked independently for her doctoral dissertation [16] on partial key recovery attacks on HAVAL-based HMAC and second preimage attack on HAVAL).

The cryptographic hash function HAVAL was proposed by Y. Zheng et al. in 1992 19. It takes an input value of arbitrary length and digests it into variant lengths of $128,160,192,224$ or 256 bits. In this paper, we present a new second preimage differential path of 3 -pass HAVAL with probability $2^{-114}$ and devise a second preimage attack on 3-pass HAVAL, and a partial key recovery attack on HMAC/NMAC-3-pass HAVAL with $2^{122}$ oracle queries, $5 \cdot 2^{32}$ memory bytes and $2^{96} 3$-pass HAVAL computations.

This paper is organized as follows. In Section 2, we describe HAVAL, HMAC, NMAC, and notations. Next, we present a second preimage attack on 3-pass HAVAL in Section 3 and apply it to recover a partial key of HMAC/NMAC-3pass HAVAL in Section 4. Finally, we conclude in Section 5.

\section{Preliminaries}

In this section, we give a brief description of the HAVAL hash function, the HMAC/NMAC algorithms and notations used in the paper.

\subsection{Description of HAVAL}

HAVAL produces hashes in different lengths of 128, 160, 192, 224 and 256 bits. It allows that users can choose the number of passes 3,4 or 5 , where each pass contains 32 steps. It computes the hashes in the following procedure:

- Padding: an inserted message is padded into a multiple of 1024 bits.

- Compression function $H$ : let $M^{0}, M^{1}, \cdots, M^{S}$ be 1024-bit message blocks and each $M^{i}$ consists of 32 32-bit words, that is, $M^{i}=M_{0}^{i}\left\|M_{1}^{i}\right\| \cdots \| M_{31}^{i}$, where $M_{j}^{i}$ is a 32 -bit word.

- $h_{0}=H\left(I V, M^{0}\right)$, where $I V$ is the initial value.

- $h_{1}=H\left(h_{0}, M^{1}\right), \cdots, h_{s}=H\left(h_{s-1}, M^{S}\right)$

- Output of HAVAL: $H_{n}$

The HAVAL compression function $H$ processes 3,4 or 5 passes. Let $F_{1}, F_{2}, F_{3}$, $F_{4}$ and $F_{5}$ be the five passes and $\left(D_{i n}, M\right)$ be the input value of $H$, where $D_{i n}$ is a 256-bit initial block and $M$ is a 1024-bit message block. Then the output of the compression function $D_{\text {out }}$ can be computed in the following way.

$$
\begin{array}{r}
E_{0}=D_{i n}, E_{1}=F_{1}\left(E_{0}, M\right), E_{2}=F_{2}\left(E_{1}, M\right), E_{3}=F_{3}\left(E_{2}, M\right) ; \\
E_{4}=F_{4}\left(E_{3}, M\right) \quad(\text { pass }=4,5), E_{5}=F_{5}\left(E_{4}, M\right) \quad(\text { pass }=5) ;
\end{array}
$$




$$
D_{\text {out }}= \begin{cases}E_{3} \boxplus E_{0}, & \text { pass }=3 \\ E_{4} \boxplus E_{0}, & \text { pass }=4 \\ E_{5} \boxplus E_{0}, & \text { pass }=5\end{cases}
$$

Fig. 1 shows the $i$-th step of HAVAL, where $a_{i}$ represents the updated 32bit value of the $i$-th step. Let a 1024-bit message block $M$ be denoted $M=$ $M_{0}\left\|M_{1}\right\| \cdots\left\|M_{30}\right\| M_{31}$, where $M_{i}(i=0,1, \cdots, 31)$ is a 32 -bit word, then the orders of the message words in each pass are as in Table 1 .

Each pass employs a different Boolean function $f_{i}(i=1,2,3,4,5)$ and a different permutation function. The following $f_{i}$ is used in pass $i$ :

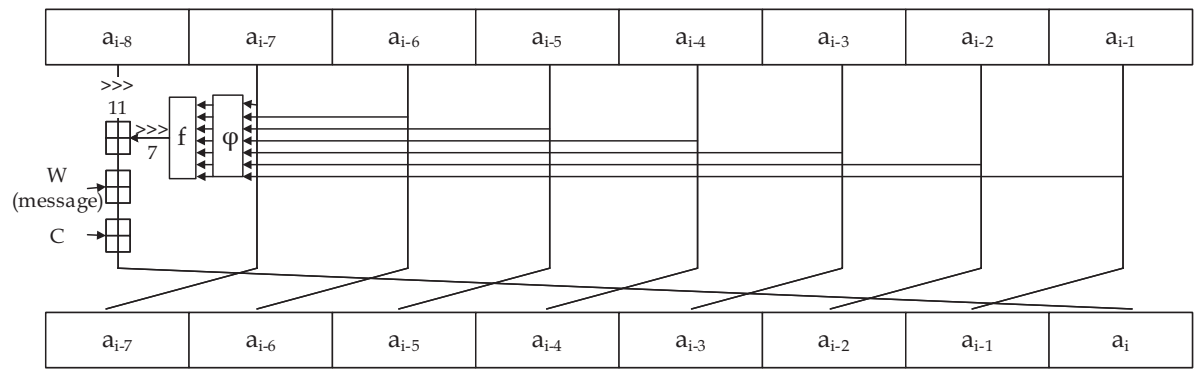

Fig. 1. $i$-th step of HAVAL hash function

Table 1. Orders of message words

\begin{tabular}{|c|c|}
\hline Pa. & \begin{tabular}{|cccccccccccccccc}
0 & 1 & 2 & 3 & 4 & 5 & 6 & 7 & 8 & 9 & 10 & 11 & 12 & 13 & 14 & 15 \\
16 & 17 & 18 & 19 & 20 & 21 & 22 & 23 & 24 & 25 & 26 & 27 & 28 & 29 & 30 & 31
\end{tabular} \\
\hline & \begin{tabular}{|ccccccccccccccccc}
5 & 14 & 26 & 18 & 11 & 28 & 7 & 16 & 0 & 23 & 20 & 22 & 1 & 10 & 4 & 8 \\
30 & 3 & 21 & 9 & 17 & 24 & 29 & 6 & 19 & 12 & 15 & 13 & 2 & 25 & 31 & 2
\end{tabular} \\
\hline & $\mid \begin{array}{ccccccccccccccccc}19 & 9 & 4 & 20 & 28 & 17 & 8 & 22 & 29 & 14 & 25 & 12 & 24 & 30 & 16 & 26 \\
31 & 15 & 7 & 3 & 1 & 0 & 28 & 27 & 13 & 6 & 21 & 10 & 23 & 11 & 5 & 2\end{array}$ \\
\hline & \begin{tabular}{|ccccccccccccccccc}
24 & 4 & 0 & 14 & 2 & 7 & 28 & 23 & 26 & 6 & 30 & 20 & 18 & 25 & 19 & 3 \\
22 & 11 & 31 & 21 & 8 & 27 & 12 & 9 & 1 & 29 & 5 & 15 & 17 & 10 & 16 & 13 \\
\end{tabular} \\
\hline & $\begin{array}{|ccccccccccccccc|}27 & 3 & 21 & 26 & 17 & 11 & 20 & 29 & 19 & 0 & 12 & 7 & 13 & 8 & 31 \\
5 & 9 & 14 & 30 & 18 & 6 & 28 & 24 & 2 & 23 & 16 & 22 & 4 & 1 & 25 \\
\end{array}$ \\
\hline
\end{tabular}

$f_{1}\left(x_{6}, x_{5}, x_{4}, x_{3}, x_{2}, x_{1}, x_{0}\right)=x_{1} x_{4} \oplus x_{2} x_{5} \oplus x_{3} x_{6} \oplus x_{0} x_{1} \oplus x_{0}$ $f_{2}\left(x_{6}, x_{5}, x_{4}, x_{3}, x_{2}, x_{1}, x_{0}\right)=x_{1} x_{2} x_{3} \oplus x_{2} x_{4} x_{5} \oplus x_{1} x_{2} \oplus x_{1} x_{4} \oplus$ $x_{2} x_{6} \oplus x_{3} x_{5} \oplus x_{4} x_{5} \oplus x_{0} x_{2} \oplus x_{0}$ $f_{3}\left(x_{6}, x_{5}, x_{4}, x_{3}, x_{2}, x_{1}, x_{0}\right)=x_{1} x_{2} x_{3} \oplus x_{1} x_{4} \oplus x_{2} x_{5} \oplus x_{3} x_{6} \oplus x_{0} x_{3} \oplus x_{0}$ $f_{4}\left(x_{6}, x_{5}, x_{4}, x_{3}, x_{2}, x_{1}, x_{0}\right)=x_{1} x_{2} x_{3} \oplus x_{2} x_{4} x_{5} \oplus x_{3} x_{4} x_{6} \oplus x_{1} x_{4} \oplus x_{2} x_{6} \oplus$ $x_{3} x_{4} \oplus x_{3} x_{5} \oplus x_{3} x_{6} \oplus x_{4} x_{5} \oplus x_{4} x_{6} \oplus x_{0} x_{4} \oplus x_{0}$ $f_{5}\left(x_{6}, x_{5}, x_{4}, x_{3}, x_{2}, x_{1}, x_{0}\right)=x_{1} x_{4} \oplus x_{2} x_{5} \oplus x_{3} x_{6} \oplus x_{0} x_{1} x_{2} x_{3} \oplus x_{0} x_{5} \oplus x_{0}$ 
Let $\varphi_{i, j}$ be the permutation function of the $j$-th pass of the $i$-pass HAVAL. Table 2 shows the $\varphi_{i, j}$ used in each pass. In each step, the updated value $a_{i}$ is computed as

$$
a_{i}=\left(a_{i-8} \ggg 11\right) \boxplus\left(f\left(\varphi\left(a_{i-7}, a_{i-6}, \cdots, a_{i-1}\right)\right) \ggg 7\right) \boxplus M_{i} \boxplus C,
$$

where $X \ggg i$ is the right cyclic rotation of $X$ by $i$ bits, and $C$ is a constant.

Table 2. $\varphi_{i, j}$ used in each pass

\begin{tabular}{|c|c|}
\hline permutations & $\begin{array}{lllllll}x_{6} & x_{5} & x_{4} & x_{3} & x_{2} & x_{1} & x_{0}\end{array}$ \\
\hline$\varphi_{3,1}$ & $\begin{array}{lllllll}x_{1} & x_{0} & x_{3} & x_{5} & x_{6} & x_{2} & x_{4}\end{array}$ \\
\hline$\varphi_{3,2}$ & $\begin{array}{lllllll}x_{4} & x_{2} & x_{1} & x_{0} & x_{5} & x_{3} & x_{6} \\
\end{array}$ \\
\hline$\varphi_{3,3}$ & $\begin{array}{lllllll}x_{6} & x_{1} & x_{2} & x_{3} & x_{4} & x_{5} & x_{0}\end{array}$ \\
\hline$\varphi_{4,1}$ & $\begin{array}{lllllll}x_{2} & x_{6} & x_{1} & x_{4} & x_{5} & x_{3} & x_{0}\end{array}$ \\
\hline$\varphi_{4,2}$ & $\begin{array}{lllllll}x_{3} & x_{5} & x_{2} & x_{0} & x_{1} & x_{6} & x_{4} \\
\end{array}$ \\
\hline$\varphi_{4,3}$ & $\begin{array}{lllllll}x_{1} & x_{4} & x_{3} & x_{6} & x_{0} & x_{2} & x_{5}\end{array}$ \\
\hline$\varphi_{4,4}$ & $\begin{array}{lllllll}x_{6} & x_{4} & x_{0} & x_{5} & x_{2} & x_{1} & x_{3}\end{array}$ \\
\hline$\varphi_{5,1}$ & $\begin{array}{llllllll}x_{3} & x_{4} & x_{1} & x_{0} & x_{5} & x_{2} & x_{6}\end{array}$ \\
\hline$\varphi_{5,2}$ & $\begin{array}{lllllll}x_{6} & x_{2} & x_{1} & x_{0} & x_{3} & x_{4} & x_{5}\end{array}$ \\
\hline$\varphi_{5,3}$ & $\begin{array}{lllllll}x_{2} & x_{6} & x_{0} & x_{4} & x_{3} & x_{1} & x_{5}\end{array}$ \\
\hline$\varphi_{5,4}$ & $\begin{array}{lllllll}x_{1} & x_{5} & x_{3} & x_{2} & x_{0} & x_{4} & x_{6}\end{array}$ \\
\hline$\varphi_{5,5}$ & $\begin{array}{lllllll}x_{2} & x_{5} & x_{0} & x_{6} & x_{4} & x_{3} & x_{1}\end{array}$ \\
\hline
\end{tabular}

\subsection{Description of HMAC/NMAC}

Fig. 2] shows NMAC and HMAC based on a compression function $f$ which maps $\{0,1\}^{n} \times\{0,1\}^{b}$ to $\{0,1\}^{n}$. The $K_{1}$ and $K_{2}$ are all $n$-bit keys and the $\bar{K}=$ $K \| 0^{b-n}$, where $K$ is an $n$-bit key. The opad is formed by repeating the byte ' $0 \times 36$ ' as many times as needed to get a $b$-bit block, and the ipad is defined similarly using the byte ' $0 \times 5 c$ '.

Let $F:\{I V\} \times\left(\{0,1\}^{b}\right)^{*} \rightarrow\{0,1\}^{n}$ be the iterated hash function defined as $F\left(I V, M^{1}\left\|M^{2}\right\| \cdots \| M^{S}\right)=f\left(\cdots f\left(f\left(I V, M^{1}\right), M^{2}\right) \cdots, M^{S}\right)$, where $M^{i}$ is a $b$ bit message. Let $g$ be a padding method, $g(x)=x\left\|10^{t}\right\| \operatorname{bin}_{64}(x)$, where $t$ is the smallest non-negative integer such that $g(x)$ is a multiple of $b$ and $\operatorname{bin}_{i}(x)$ is the $i$-bit binary representation of $x$. Then, NMAC and HMAC are defined as follows:

$$
\begin{aligned}
\operatorname{NMAC}_{K_{1}, K_{2}}(M) & =H\left(K_{2}, g\left(H\left(K_{1}, g(M)\right)\right)\right) \\
\operatorname{HMAC}_{K}(M) & =H(I V, g(\bar{K} \oplus \text { opad }|| H(I V, g(\bar{K} \oplus \text { ipad } \| M)))) .
\end{aligned}
$$

\subsection{Notations}

Let $M$ and $M^{\prime}$ be 1024-bit messages such that $M=M_{0}\left\|M_{1}\right\| \cdots \| M_{31}$ and $M^{\prime}=$ $M_{0}^{\prime}\left\|M_{1}^{\prime}\right\| \cdots \| M_{31}^{\prime}$, where $M_{i}(i=0,1,2, \cdots, 31)$ and $M_{j}^{\prime}(j=0,1,2, \cdots, 31)$ are 32 -bit words. We denote by $a_{i}$ (resp., $a_{i}^{\prime}$ ) the updated value of the $i$-th step using 

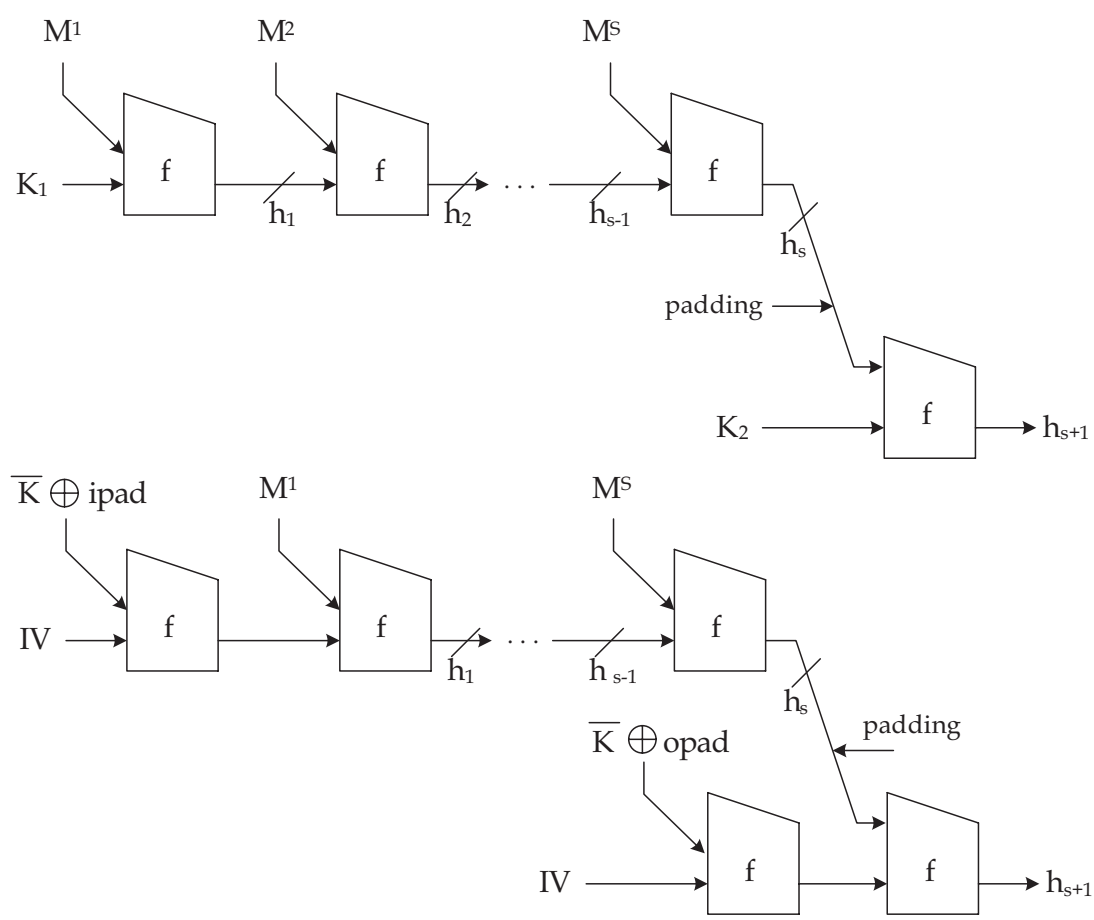

Fig. 2. NMAC and HMAC

the message $M$ (resp., $M^{\prime}$ ). Let $t_{i}$ (resp., $t_{i}^{\prime}$ ) be the output value of the Boolean function of the $i$-th step using the message $M$ (resp., $M^{\prime}$ ). The $j$-th bits of $a_{i}$ and $t_{i}$ are denoted $a_{i, j}$ and $t_{i, j}$. Additionally, we use several following notations in our attacks, where $0 \leq j \leq 31$.

$-a_{i}[j]: a_{i, j}=0, a_{i, j}^{\prime}=1$,

$-a_{i}[-j]: a_{i, j}=1, a_{i, j}^{\prime}=0$,

$-t_{i}[j]: t_{i, j}=0, t_{i, j}^{\prime}=1$,

$-t_{i}[-j]: t_{i, j}=1, t_{i, j}^{\prime}=0$.

\section{Second Preimage Attack on 3-Pass HAVAL}

In this section, we show how to construct a second preimage differential path of 3-pass HAVAL. Using this differential path, we find a second preimage of 3pass HAVAL with probability $2^{-114}$, i.e., for a given message $M$, we find another message $M^{\prime}$ with probability $2^{-114}$ satisfying $H(M)=H\left(M^{\prime}\right)$, where $H$ is 3 -pass HAVAL. Our differential path of 3-pass HAVAL is stronger than the previous ones [7/9 11/12] against the second preimage attack. 


\subsection{Second Preimage Differential Path of 3-Pass HAVAL}

Let two 1024-bit message blocks $M=M_{0}\left\|M_{1}\right\| M_{2}\|\cdots\| M_{31}$ and $M^{\prime}=M_{0}^{\prime}\left\|M_{1}^{\prime}\right\|$ $M_{2}^{\prime}\|\cdots\| M_{31}^{\prime}$ satisfy $M_{i}=M_{i}^{\prime}$ for $i=0,1, \cdots, 21,23,24, \cdots, 31$ and $M_{22} \oplus$ $M_{22}^{\prime}=2^{31}$. Then we can use these two messages to construct a second preimage differential path of 3 -pass HAVAL with probability $2^{-114}$. Table 3 shows our second preimage differential path of 3-pass HAVAL, which has been constructed as follows.

First of all, from the message pair we get the input difference to the 23-rd step $\left(\Delta a_{15}, \Delta a_{16}, \Delta a_{17}, \Delta a_{18}, \Delta a_{19}, \Delta a_{20}, \Delta a_{21}, \Delta a_{22}\right)=\left(0,0,0,0,0,0,0, a_{22}[31]\right)$ if a condition $a_{22,31}=0$ holds. Recall that $\left(a_{i-8}, a_{i-7}, \cdots, a_{i-2}, a_{i-1}\right)$ is the input state to the $i$-th step. We assume that the output differences of the Boolean functions from the 23-rd step to the 36 -th step are all zeroes. Then we can obtain the input difference to the 37 -th step is $\left(0, a_{30}[20], 0,0,0,0,0,0\right)$. It is easy to see that the required assumption works if several conditions hold in our differential, which we call sufficient conditions. For example, consider a difference $\Delta t_{24}$. The input difference to the 24-th step is $\left(\Delta a_{16}, \Delta a_{17}, \Delta a_{18}, \Delta a_{19}, \Delta a_{20}, \Delta a_{21}, \Delta a_{22}, \Delta a_{23}\right)=$ $\left(0,0,0,0,0,0, a_{22}[31], 0\right)$. The permutation is $\varphi\left(x_{6}, x_{5}, x_{4}, x_{3}, x_{2}, x_{1}, x_{0}\right)=\left(x_{1}, x_{0}\right.$, $\left.x_{3}, x_{5}, x_{6}, x_{2}, x_{4}\right)$ and the Boolean function is $f\left(x_{6}, x_{5}, x_{4}, x_{3}, x_{2}, x_{1}, x_{0}\right)=x_{1} x_{4} \oplus$ $x_{2} x_{5} \oplus x_{3} x_{6} \oplus x_{0} x_{1} \oplus x_{0}$ in the 24-th step. Thus, $f\left(\varphi\left(x_{6}, x_{5}, x_{4}, x_{3}, x_{2}, x_{1}, x_{0}\right)\right)$ $=x_{2} x_{3} \oplus x_{6} x_{0} \oplus x_{5} x_{1} \oplus x_{4} x_{2} \oplus x_{4}$ and the most significant bit of the output of the Boolean function in the 24-th step is $a_{20,31} a_{21,31} \oplus a_{17,31} a_{23,31} \oplus a_{18,31} a_{22,31} \oplus$ $a_{19,31} a_{21,31} \oplus a_{19,31}$. If $a_{18,31}=0$, then the difference of $a_{22,31}$ does not have effect on the output difference of the Boolean function and thus $\Delta t_{24}=0$. Thus, $a_{18,31}=0$ is one of the sufficient conditions. We show in Table 5 of appendix all the sufficient conditions which satisfy our differential path.

In order to compute the probability that a message $M$ satisfies the sufficient conditions listed in Table 5, we need to check the dependency of the conditions. To make the problem easier we first solve and simplify the conditions. In this process we may reduce the number of the sufficient conditions. Consider the conditions on the 20-th bit from the 31-st step to the 37-th step in Table 5 .

1. 31-st step : $a_{30,20}=0, a_{24,20}=0$

2. 32-nd step : $a_{29,20} a_{26,20} \oplus a_{28,20} \oplus a_{29,20}=0$

3. 33-rd step : $a_{31,20} a_{27,20} \oplus a_{32,20} \oplus a_{31,20}=0$

4. 34-th step : $a_{33,20} a_{28,20} \oplus a_{28,20} \oplus a_{32,20}=0$

5. 35 -th step : $a_{29,20}=0$

6. 36-th step : $a_{35,20} a_{32,20} \oplus a_{34,20} a_{33,20} \oplus a_{32,20} \oplus a_{31,20} \oplus a_{35,20}=0$

7. 37-th step : $a_{31,20}=0, a_{33,20} a_{35,20} \oplus a_{36,20} a_{34,20} \oplus a_{35,20} a_{34,20}=0$

In the 32-nd step, we can simplify the condition to $a_{28,20}=0$ by inserting the value $a_{29,20}=0$ which is the condition in the 35 -th step. Using this condition $a_{28,20}=0$, we can obtain $a_{32,20}=0$ in the 34 -th step. This simplified condition $a_{32,20}=0$ and the 37-th step condition $a_{31,20}=0$ make the 33-rd step condition always hold. Moreover, the 36 -th step condition is simplified to $a_{34,20} a_{33,20} \oplus a_{35,20}=0$ due to the conditions $a_{31,20}=0$ and $a_{32,20}=0$. Following is the simplified conditions for steps 31-37 (note that the number of the sufficient conditions is reduced from 9 to 8 by solving the conditions): 
Table 3. Second preimage differential path of 3-pass HAVAL

\begin{tabular}{|c|c|c|c|c|c|c|c|c|c|c|}
\hline ep & $M_{i}$ & $\Delta t_{i}$ & $\overline{a_{i-8}}$ & $\Delta a_{i-7}$ & $\Delta a_{i-6}$ & $\Delta a_{i-5}$ & $\Delta a_{i-4}$ & $\Delta a_{i-3}$ & $\Delta a_{i-2}$ & $\Delta a_{i-1}$ \\
\hline$\overline{0}$ & $\overline{0}$ & $\overline{\overline{0}}$ & $\overline{0}$ & $\overline{00}$ & $\overline{0}$ & $\overline{0}$ & $\overline{0}$ & $\overline{0}$ & $\overline{0}$ & 0 \\
\hline$\cdots$ & $\cdots$ & $\cdots$ & & $\cdots$ & & & & & $\cdots$ & \\
\hline 21 & 0 & 0 & 0 & 0 & 0 & 0 & 0 & 0 & 0 & 0 \\
\hline 22 & \pm 31 & 0 & 0 & 0 & 0 & 0 & 0 & 0 & 0 & 0 \\
\hline 23 & 0 & 0 & 0 & 0 & 0 & 0 & 0 & 0 & 0 & 31 \\
\hline 24 & 0 & 0 & 0 & $\overline{0}$ & 0 & $\overline{0}$ & 0 & 0 & 31 & 0 \\
\hline 25 & 0 & 0 & 0 & 0 & 0 & 0 & 0 & 31 & 0 & 0 \\
\hline 26 & 0 & 0 & 0 & 0 & 0 & 0 & 31 & 0 & 0 & 0 \\
\hline 27 & 0 & 0 & 0 & 0 & 0 & 31 & 0 & 0 & 0 & 0 \\
\hline 28 & 0 & 0 & 0 & 0 & 31 & 0 & 0 & 0 & 0 & 0 \\
\hline 29 & 0 & 0 & 0 & 31 & 0 & 0 & 0 & 0 & 0 & 0 \\
\hline 30 & 0 & 0 & 31 & 0 & 0 & 0 & 0 & 0 & 0 & 0 \\
\hline 31 & 0 & 0 & 0 & 0 & 0 & 0 & 0 & 0 & 0 & 20 \\
\hline 32 & 0 & 0 & 0 & 0 & 0 & 0 & 0 & 0 & 20 & 0 \\
\hline 33 & 0 & 0 & 0 & 0 & 0 & 0 & 0 & 20 & 0 & 0 \\
\hline 34 & 0 & 0 & 0 & 0 & 0 & 0 & 20 & 0 & 0 & 0 \\
\hline 35 & 0 & 0 & 0 & 0 & 0 & 20 & 0 & 0 & 0 & 0 \\
\hline 36 & 0 & 0 & 0 & 0 & 20 & 0 & 0 & 0 & 0 & 0 \\
\hline 37 & 0 & 20 & 0 & 20 & 0 & 0 & 0 & 0 & 0 & 0 \\
\hline 38 & 0 & 0 & 20 & 0 & 0 & 0 & 0 & 0 & 0 & 13 \\
\hline 39 & 0 & 0 & 0 & 0 & 0 & 0 & 0 & 0 & 13 & 9 \\
\hline 40 & 0 & 0 & 0 & 0 & 0 & 0 & 0 & 13 & 9 & 0 \\
\hline 41 & 0 & 0 & 0 & 0 & 0 & 0 & 13 & 9 & 0 & 0 \\
\hline 42 & 0 & 13 & 0 & 0 & 0 & 13 & 9 & 0 & 0 & 0 \\
\hline 43 & \pm 31 & \pm 6 & 0 & 0 & 13 & 9 & 0 & 0 & 0 & 6 \\
\hline 44 & 0 & 0 & 0 & 13 & 9 & 0 & 0 & 0 & 6 & 0 \\
\hline 45 & 0 & -9 & 13 & 9 & 0 & 0 & 0 & 6 & 0 & 0 \\
\hline 46 & 0 & 0 & 9 & 0 & 0 & 0 & 6 & 0 & 0 & 0 \\
\hline 47 & 0 & 0 & 0 & 0 & 0 & 6 & 0 & 0 & 0 & 30 \\
\hline 48 & 0 & 0 & 0 & 0 & 6 & 0 & 0 & 0 & 30 & 0 \\
\hline 49 & 0 & 0 & 0 & 6 & 0 & 0 & 0 & 30 & 0 & 0 \\
\hline 50 & 0 & 0 & 6 & 0 & 0 & 0 & 30 & 0 & 0 & 0 \\
\hline 51 & 0 & 0 & 0 & 0 & 0 & 30 & 0 & 0 & 0 & $-27,28$ \\
\hline 52 & 0 & 0 & 0 & 0 & 30 & 0 & 0 & 0 & $-27,28$ & 0 \\
\hline 53 & 0 & 30 & 0 & 30 & 0 & 0 & 0 & $-27,28$ & 0 & 0 \\
\hline 54 & 0 & 0 & 30 & 0 & 0 & 0 & $-27,28$ & 0 & 0 & 23 \\
\hline 55 & 0 & 28 & 0 & 0 & 0 & $-27,28$ & 0 & 0 & 23 & 19 \\
\hline 56 & 0 & 21 & 0 & 0 & $-27,28$ & 0 & 0 & 23 & 19 & 21 \\
\hline 57 & 0 & 0 & 0 & $-27,28$ & 0 & 0 & 23 & 19 & 21 & $-14,15$ \\
\hline 58 & 0 & \begin{tabular}{|l|}
-23 \\
\end{tabular} & $-27,28$ & 0 & 0 & 23 & 19 & 21 & $-14,15$ & 0 \\
\hline 59 & 0 & 0 & 0 & 0 & 23 & 19 & 21 & $-14,15$ & 0 & 0 \\
\hline 60 & 0 & $\overline{0}$ & 0 & 23 & 19 & 21 & $-14,15$ & 0 & 0 & 0 \\
\hline 61 & 0 & \begin{tabular}{|l|}
-19 \\
\end{tabular} & 23 & 19 & 21 & $-14,15$ & 0 & 0 & 0 & 0 \\
\hline 62 & 0 & -15 & 19 & 21 & $-14,15$ & 0 & 0 & 0 & 0 & 0 \\
\hline 63 & 0 & 0 & 21 & $-14,15$ & 0 & 0 & 0 & 0 & 0 & 0 \\
\hline 64 & 0 & -10 & $-14,15$ & 0 & 0 & 0 & 0 & 0 & 0 & 10 \\
\hline 65 & 0 & 0 & 0 & 0 & 0 & 0 & 0 & 0 & 10 & 0 \\
\hline 66 & 0 & 0 & 0 & 0 & 0 & 0 & 0 & 10 & 0 & 0 \\
\hline 67 & 0 & 0 & 0 & 0 & 0 & 0 & 10 & 0 & 0 & 0 \\
\hline 68 & 0 & 0 & 0 & 0 & 0 & 10 & 0 & 0 & 0 & 0 \\
\hline 69 & 0 & 0 & 0 & 0 & 10 & 0 & 0 & 0 & 0 & 0 \\
\hline 70 & 0 & 0 & 0 & 10 & 0 & 0 & 0 & 0 & 0 & 0 \\
\hline 71 & \pm 31 & 0 & 10 & 0 & 0 & 0 & 0 & 0 & 0 & 0 \\
\hline \begin{tabular}{|l|}
72 \\
\end{tabular} & 0 & 0 & 0 & 0 & 0 & 0 & 0 & 0 & 0 & 0 \\
\hline$\cdots$ & $\cdots$ & $\cdots$ & & $\cdots$ & & & & & $\cdots$ & \\
\hline 95 & 0 & 0 & 0 & 0 & 0 & 0 & 0 & 0 & 0 & 0 \\
\hline
\end{tabular}


1. 31-st step : $a_{30,20}=0, a_{24,20}=0$

2. 32 -nd step : $a_{28,20}=0$

3. 33-rd step : no condition

4. 34-th step : $a_{32,20}=0$

5. 35-th step : $a_{29,20}=0$

6. 36-th step : $a_{34,20} a_{33,20} \oplus a_{35,20}=0$

7. 37-th step : $a_{31,20}=0, a_{33,20} a_{35,20} \oplus a_{36,20} a_{34,20} \oplus a_{35,20} a_{34,20}=0$

Table 6] in appendix collects all the simplified conditions for those of Table 5. We notice that the number of the sufficient conditions listed in Table 6 is 112, which seems to make the probability that a message satisfy all these conditions is $2^{-112}$. However, it is not $2^{-112}$, but approximately $2^{-114}$. This is due to the fact that there are still dependencies in some conditions. For example, consider the conditions on the 13 -th bit from the 38 -th step to the 41 -st step in Table 6 .

1. 38-th step : $a_{38,13}=1, a_{34,13} a_{32,13} \oplus a_{35,13}=0$

2. 39-th step : $a_{33,13} \neq a_{35,13}$

3. 40-th step : $a_{34,13} \neq a_{39,13}$

4. 41-st step : $a_{40,13} a_{35,13} \oplus a_{35,13} \oplus a_{39,13}=1$

These 5 conditions do not hold with probability $2^{-5}$, but with probability $2^{-3} \cdot \frac{3}{16}$. The reason is as follows. The probability that the condition $a_{38,13}=1$ is satisfied is $2^{-1}$. Table 4 lists all the possible values of $a_{32,13}, a_{34,13}$ and $a_{35,13}$ which satisfy $a_{34,13} a_{32,13} \oplus a_{35,13}=0$. The probability that this condition holds is $\frac{1}{2}\left(=\frac{4}{8}\right)$ according to Table 4 . In the 39 -th step, the probability that $a_{33,13} \neq a_{35,13}$ is satisfied is $2^{-1}$ since $a_{33,13}$ is used only in the 39 -th step. In the 40-th and 41-st steps, if $a_{35,13}=0$, then $a_{39,13}$ and $a_{34,13}$ should be 0 and 1 , respectively, and $a_{40,13}$ is either 0 or 1 . The probability that $a_{35,13}=0$ and $a_{34,13}=1$ hold is $\frac{1}{4}$ (one out of four cases, see Table 4). Thus the probability that $a_{34,13}=1, a_{35,13}=0$, and $a_{39,13}=0$ are satisfied is $\frac{1}{8}\left(=\frac{1}{4} \cdot \frac{1}{2}\right)$ (recall that $a_{40,13}$ does not have effect on the condition $\left.a_{40,13} a_{35,13} \oplus a_{35,13} \oplus a_{39,13}=1\right)$. If $a_{35,13}=1$ and $a_{39,13}=1$, then $a_{40,13}=1$ and $a_{34,13}=0$ due to the conditions $a_{40,13} a_{35,13} \oplus a_{35,13} \oplus a_{39,13}=1$ and $a_{34,13} \neq a_{39,13}$. However, this is a contradiction to the condition of the 38th step (see Table 4), and thus if $a_{35,13}=1$, then $a_{39,13}=0, a_{40,13}=1$ and $a_{34,13}=1$. The probability that $a_{35,13}=1$ and $a_{34,13}=1$ hold is $\frac{1}{4}$ by Table 4 and each probability of $a_{39,13}=0$ and $a_{40,13}=1$ is $\frac{1}{2}$, so the probability that $\left(a_{34,13}, a_{35,13}, a_{39,13}, a_{40,13}\right)=(1,1,0,1)$ is $\frac{1}{16}$. Therefore, we can compute the probability that the conditions in the 40 -th and 41-st step hold is $\frac{3}{16}\left(=\frac{1}{8}+\frac{1}{16}\right)$, leading to a total probability $2^{-3} \cdot \frac{3}{16}$ for the above 5 conditions. In this way, we analyze the probability that the sufficient conditions in Table 6 are satisfied is $2^{-114}$.

\subsection{Attack on 3-Pass HAVAL}

The second preimage resistance on a hash function plays an important role to block the attacker to produce a second preimage when a meaningful and sensitive message (e.g. a finance-related message) is used. In literature, it is defined as follows: 
Table 4. Possible values for the conditions on the 38-th, 40-th and 41-st step

\begin{tabular}{|l|c|c|c|c|c|}
\hline step & & $a_{32,13}$ & $a_{34,13}$ & $a_{35,13}$ & probability \\
\hline \multirow{3}{*}{38} & & 1 & 1 & 1 & $1 / 8$ \\
& & 0 & 1 & 0 & $1 / 8$ \\
& & 1 & 0 & 0 & $1 / 8$ \\
& & 0 & 0 & 0 & $1 / 8$ \\
\hline \hline step & $a_{34,13}$ & $a_{35,13}$ & $a_{39,13}$ & $a_{40,13}$ & probability \\
\hline \multirow{2}{*}{40,} & 1 & 0 & 0 & 0 & $1 / 4 \times 1 / 2 \times 1 / 2$ \\
41 & 1 & 0 & 0 & 1 & $1 / 4 \times 1 / 2 \times 1 / 2$ \\
& 1 & 1 & 0 & 1 & $1 / 4 \times 1 / 2 \times 1 / 2$ \\
\hline
\end{tabular}

Second preimage resistance on a hash function $\mathbf{H}$. for any given message $M$, it is computationally infeasible to find another message $M^{\prime}$ satisfying $H(M)=H\left(M^{\prime}\right)$

It follows that the second preimage attack on a hash function exists if for a given message $M$ there is an algorithm that finds another message $M^{\prime}$ such that $H(M)=H\left(M^{\prime}\right)$ with probability larger than $2^{-n}$, where $n$ is the bit-length of hash values. The second preimage attack on 3-pass HAVAL works due to our differential path;

- For a given message $M$, the probability that $M$ holds the sufficient conditions listed in Table 6 is $2^{-114}$.

- If the message $M$ holds the sufficient conditions, then the message $M^{\prime}$ which only differs from $M$ at the most significant bit of the 22-nd message word has a same hash value.

\section{Partial Key-Recovery Attacks on HMAC/NMAC-3- Pass HAVAL}

In this section, we present partial key recovery attacks on HMAC/NMAC-3pass HAVAL, which works based on our differential path described in Section 3. More precisely, we show how to find the partial key $K_{1}$ of NMAC-3-pass HAVAL and $f(\bar{K} \oplus$ ipad) of HMAC-3-pass HAVAL (note that knowing $f(\bar{K} \oplus$ ipad) and $f(\bar{K} \oplus$ opad $)$ allows to compute the MAC value for any message). Since HMAC $=$ NMAC if $f(\bar{K} \oplus$ ipad $)=K_{1}$ and $f(\bar{K} \oplus$ opad $)=K_{2}$, we focus on the NMAC-3pass HAVAL attack which finds $K_{1}$ with message/MAC pairs. Recall that $K_{1}$ is placed at the position of the initial state in NMAC. This implies that recovering the initial value of 3 -pass HAVAL is equivalent to getting the partial key $K_{1}$ of NMAC-3-pass HAVAL.

The main idea behind of our attack is that the attacker can recover the initial state of NMAC-3-pass HAVAL (in our attack it is $K_{1}$ ) if he knows a 256-bit input value at any step of 3 -pass HAVAL. This idea has firstly been introduced in [4. In this section, we first find $a_{16}, a_{18}, a_{21}$ and $a_{23}$ which are used as a part of an input value to the 24 -th step. Remaining four-word input values $a_{17}, a_{19}, a_{20}$ 
and $a_{22}$ to the 24-th step is then found by $2^{128}$ exhaustive searches. Let $a_{i, j}$ be the $j$-th bit of $a_{i}$ and $\gamma_{i}=\left(a_{i-8} \ggg 11\right) \boxplus\left(t_{i} \ggg 7\right) \boxplus C$, where $C$ is a constant used in step $i$ (note $\gamma_{i} \boxplus M_{i}=a_{i}$ ).

The value $a_{16}$ is then revealed by the following Algorithm.

Algorithm 1. In order to recover the value $a_{16}$, we use a condition $a_{16,31}=0$ depicted in Table 6. The procedure goes as follows:

1. The attacker has access to the oracle $\mathcal{O}(=$ NMAC-3-pass HAVAL $)$ and makes $2^{121}$ queries for $2^{120}$ message pairs $M=M_{0}, M_{1}, \cdots, M_{30}, M_{31}$ and $M^{\prime}=$ $M_{0}^{\prime}, M_{1}^{\prime}, \cdots, M_{30}^{\prime}, M_{31}^{\prime}$ that have the message difference given in Table 5 . Among the $2^{120}$ message pairs, $M_{0}, M_{1}, \cdots, M_{15}$ and $M_{0}^{\prime}, M_{1}^{\prime}, \cdots, M_{15}^{\prime}$ are all identically fixed, $M_{16}$ and $M_{16}^{\prime}$ vary in all $2^{32}$ possible values, and $2^{88}$ message pairs in the remaining words $M_{17}, M_{18}, \cdots, M_{31}$ and $M_{17}^{\prime}, M_{18}^{\prime}, \cdots, M_{31}^{\prime}$ are randomly chosen. In this case, what the attacker knows is that $\gamma_{16}$ is identically fixed for all the $2^{120}$ message pairs even though he does not know the actual value $\gamma_{16}$.

2. For each candidate value $\gamma_{16}$ in $0,1, \cdots, 2^{32}-1$;

(a) Choose the message pairs $\left(M, M^{\prime}\right)$ that make collisions for the corresponding MAC pairs.

(b) Count the number of the message pairs chosen in Step 2(a) that satisfy $m s b\left(\gamma_{16} \boxplus M_{16}\right)=1$.

3. Output $\gamma_{16} \boxplus M_{16}$ as $a_{16}$, where $\gamma_{16}$ has the least count number in Step 2 (b).

As mentioned before, this algorithm works due to our differential with probability $2^{-114}$. Notice that our differential encompasses a sufficient condition $a_{16,31}=0$, and each message pair among the $2^{120}$ message pairs satisfies the condition $a_{16,31}=0$, our differential holds with probability $2^{-113}$ with respect to this message pair. If the message pair $\left(M, M^{\prime}\right)$ makes the most significant bits of $a_{16}$ and $a_{16}^{\prime}$ be 1 , then the probability that the message pair $\left(M, M^{\prime}\right)$ makes a collision is $2^{-121}\left(=2^{-113} \cdot 2^{-8}\right)$, for it forces additionally 8 more sufficient conditions in our collision producing differential. The reason is as follows. If $a_{16,31}=1$, then a difference $\Delta t_{23}$ is not zero, but $\pm 2^{31}$. However, this difference value can be canceled by the output difference of the Boolean function in the $31-$ st step. In this procedure, each of steps 24-31 requires one more additional condition, leading to total 8 additional conditions. Thus, the probability that the message pair $\left(M, M^{\prime}\right)$ has a same MAC value is not a random probability but $2^{-121}$, where the most significant bits of $a_{16}$ and $a_{16}^{\prime}$ are 1 . It follows that if the right $\gamma_{16}$ is guessed, we expect $2^{-2}\left(=2^{119} \cdot 2^{-121}\right)$ collision pairs. On the other hand, if $\gamma_{16}$ is wrongly guessed, the expectation of collision pairs is $2^{5}\left(=2^{118} \cdot 2^{-113}+2^{118} \cdot 2^{-121}\right)$, (note that in the group of the message pairs such that $m s b\left(\gamma_{16} \boxplus M_{16}\right)=1$ there are on average half message pairs satisfying the actual $\left.a_{16,31}=0\right)$. Since the probability that a wrong $\gamma_{16}$ does not cause any collision pair is $\left(1-2^{-113}\right)^{2^{118}} \cdot\left(1-2^{-121}\right)^{2^{118}}<\left(1-2^{-113}\right)^{2^{118}}\left(\approx e^{-32}\right)<2^{-32}$, we expect that there is no wrong $\gamma_{16}$ which leads to no collision in Step 2. Hence, 
we can determine the right $\gamma_{16}$. To summarize, Algorithm 1 requires $2^{121}$ oracle queries (in Step 1) and $2^{32}$ memory bytes (the memory complexity of this attack is dominated by the counters for $\left.\gamma_{16}\right)$.

Next, we show how to recover the value $a_{18}$, for which we use the condition $a_{18,31}=0$ required in our differential. Since there is no condition on $a_{17}$ (see Table 6), the attacker chooses any message word $M_{17}$. The main idea is similar to Algorithm 1.

First of all, the attacker selects $2^{119}$ message pairs $\left(M, M^{\prime}\right)$ that have the message difference given in Table 6 , where $M_{0}, M_{1}, \cdots, M_{17}$ and $M_{0}^{\prime}, M_{1}^{\prime}, \cdots, M_{17}^{\prime}$ are all identically fixed $\left(M_{0}, M_{1}, \cdots, M_{16}\right.$ and $M_{0}^{\prime}, M_{1}^{\prime}, \cdots, M_{16}^{\prime}$ should be the same as those selected in Algorithm 1, which leads to $\left.a_{16,31}=0\right), M_{18}$ and $M_{18}^{\prime}$ vary in all $2^{32}$ possible values, and $2^{87}$ message pairs in the remaining words are randomly chosen. Once the attacker gets the corresponding MAC pairs, he performs Steps 2 and 3 of Algorithm 1 to recover $a_{18}$ by setting $\gamma_{18}, M_{18}, a_{18}$ instead of $\gamma_{16}, M_{16}$ and $a_{16}$. The reason why recovering $a_{18}$ requires half of the message pairs, compared to when recovering $a_{16}$, is that this attack algorithm exploits message pairs satisfying $a_{16,31}=0$ from the beginning. It increases by twice the probability that our differential holds. The remaining analysis is the same as that of Algorithm 1. To summarize, recovering $a_{18}$ requires $2^{120}$ oracle queries.

Next, let us see how to recover $a_{21}$. In order to recover $a_{21}$ we need to use the condition $a_{20,31}=a_{21,31}$, which is of a different form from the previous two conditions $a_{16,31}=a_{18,31}=0$. However, the core in our attack is that $a_{20,31}$ is always a same value if $M_{0}, M_{1}, \cdots, M_{20}$ and $M_{0}^{\prime}, M_{1}^{\prime}, \cdots, M_{20}^{\prime}$ are all identically fixed in all required message pairs, i.e, in $2^{118}$ message pairs (note that all these message pairs satisfy $a_{16,31}=a_{18,31}=0$, which the attacker can select from the above algorithms). Similarly, among the $2^{118}$ pairs, $M_{21}$ and $M_{21}^{\prime}$ vary in all $2^{32}$ possible values and $2^{86}$ pairs of remaining words are randomly chosen.

Algorithm 2. The attack algorithm to recover $a_{21}$ goes as follows:

1. The attacker chooses the $2^{118}$ message pairs as above and asks the oracle $\mathcal{O}$ for the corresponding $2^{118}$ MAC pairs.

2. Choose the message pairs $\left(M, M^{\prime}\right)$ that make collisions for the corresponding MAC pairs.

3. For each candidate value $\gamma_{21}$ in $0,1, \cdots, 2^{32}-1$;

(a) Divide two groups of which one contains message pairs that satisfy $m s b$ $\left(\gamma_{21} \boxplus M_{21}\right)=0$ and the other one contains message pairs that satisfy $m s b\left(\gamma_{21} \boxplus M_{21}\right)=1$.

(b) Count the number of message pairs in each group that make collisions for the corresponding MAC pairs

4. Output $\gamma_{21} \boxplus M_{21}$ as $a_{21}$, where $\gamma_{21}$ is the value that has a group containing the least count, and $M_{21}$ is the one of the values satisfying $a_{20,31}=a_{21,31}$.

If the values $a_{20}$ and $a_{21}$ satisfy the sufficient condition $a_{20,31}=a_{21,31}$, then the probability that the message pair $\left(M, M^{\prime}\right)$ makes a collision is $2^{-111}$ (note that the three conditions $a_{16,31}=a_{18,31}=0, a_{21,31}=a_{20,31}$ are excluded in the list of 
the sufficient conditions). On the other hand, if $a_{21,31} \neq a_{20,31}$, then the probability that the message pair $\left(M, M^{\prime}\right)$ makes a collision is $2^{-119}$ (similarly, 8 more conditions are additionally needed). In case the right $\gamma_{21}$ is guessed, one of the two groups is expected to have $2^{-111} \cdot 2^{117}=2^{6}$ collision pairs and the other one is expected to have $2^{-119} \cdot 2^{117}=2^{-2}$ collision pair. On the other hand, if a wrong $\gamma$ is guessed, then the both groups are expected to have $2^{-111} \cdot 2^{116}=2^{5}$ collision pairs each. It implies that the probability that a wrong $\gamma_{16}$ does not cause any collision pair is about $e^{-32}<2^{-32}$, and thus there is no wrong $\gamma_{21}$ to pass Step 3. To summarize, Algorithm 2 needs $2^{119}$ oracle queries and $2^{32}$ memory bytes. Recovering $a_{23}$ is quite similar to recovering $a_{16}$ and $a_{18}$, which requires $2^{118}$ oracle queries.

Exhaustive Search for the Remaining Four Words. Using the above algorithms, we can compute the 128 -bit $a_{16}, a_{18}, a_{21}$ and $a_{23}$ values. The remaining 128 -bit $a_{17}, a_{19}, a_{20}$ and $a_{22}$ values are found by the following algorithm. We consider a message pair $\left(M, M^{\prime}\right)$ selected from the above algorithms which makes a collision.

1. Guess a 128 -bit $a_{17}, a_{19}, a_{20}, a_{22}$ value;

(a) Check with the computed $a_{16}, a_{18}, a_{21}, a_{23}$ and the guessed $a_{17}, a_{19}, a_{20}$, $a_{22}$ that the message pair $\left(M, M^{\prime}\right)$ makes a collision. If so, we determine the guessed value as the right value. Otherwise, repeat Step 1.

(b) For the given message pair $\left(M, M^{\prime}\right)$ and $a_{16}, a_{17}, \cdots, a_{22}$, recover the initial value.

If a wrong value is guessed, the probability that it causes a collision is $2^{-256}$. Since the number of wrong $a_{17}, a_{19}, a_{20}, a_{22}$ tested in the attack is $2^{128}$ at most, we can recover the right initial value. The time complexity of the exhaustive search step is $2^{128} 3$-pass HAVAL computations.

As a result, our partial key recovery attack has $2^{121}+2^{120}+2^{119}+2^{118}=2^{121.9}$ oracle queries and $2^{128} 3$-pass HAVAL computations.

Reducing the Number of the 3-Pass HAVAL Computations. As described above, our partial key-recovery attack is completed by two phases; the first phase is to recover some portions of the 256-bit input value at step $i$, and the second is the exhaustive search phase for its remaining input bits. If we apply our attack to the input value to step 29 instead of step 24 , then we can recover $a_{21}, a_{23}, a_{24}, a_{26}$ and $a_{28}$ from the first phase with $2^{122}$ oracle queries and we recover the remaining $a_{22}, a_{25}$ and $a_{27}$ with $2^{96} 3$-pass HAVAL computations from the second phase.

\section{Conclusion}

In this paper, we have presented a new second preimage differential path of 3pass HAVAL with probability $2^{-114}$ and exploited it to devise a second preimage attack on 3-pass HAVAL, and partial key-recovery attacks on HMAC/NMAC-3pass HAVAL with $2^{122}$ oracle queries, $5 \cdot 2^{32}$ memory bytes and $2^{96} 3$-pass HAVAL computations. We expect that our attacks would be useful for the further analysis of HAVAL and HMAC/NMAC-HAVAL (e.g., full key-recovery attacks on HMAC/ NMAC-HAVAL). 


\section{References}

1. Biham, E., Chen, R.: Near-Collisions of SHA-0. In: Franklin, M. (ed.) CRYPTO 2004. LNCS, vol. 3152, pp. 290-305. Springer, Heidelberg (2004)

2. Biham, E., Chen, R., Joux, A., Carribault, P., Lemuet, C., Jalby, W.: Collisions of SHA-0 and Reduced SHA-1. In: Cramer, R.J.F. (ed.) EUROCRYPT 2005. LNCS, vol. 3494, pp. 22-35. Springer, Heidelberg (2005)

3. Boer, B.D., Bosselaers, A.: Collisions for the Compression Function of MD-5. In: Helleseth, T. (ed.) EUROCRYPT 1993. LNCS, vol. 765, pp. 293-304. Springer, Heidelberg (1994)

4. Contini, S., Yin, Y.L.: Forgery and Partial Key-Recovery Attacks on HMAC and NMAC Using Hash Collisions. In: Lai, X., Chen, K. (eds.) ASIACRYPT 2006. LNCS, vol. 4284, pp. 37-53. Springer, Heidelberg (2006)

5. Dobbertin, H.: Cryptanalsis of MD4. In: Gollmann, D. (ed.) FSE 1996. LNCS, vol. 1039, pp. 53-69. Springer, Heidelberg (1996)

6. Fouque, P.A., Leurent, G., Nguyen, P.Q.: Full Key-Recovery Attacks on HMAC/NMAC-MD4 and NMAC-MD5. In: Menezes, A. (ed.) CRYPTO 2007. LNCS, vol. 4622, pp. 13-30. Springer, Heidelberg (2007)

7. Kim, J., Biryukov, A., Preneel, B., Hong, S.: On the Security of HMAC and NMAC Based on HAVAL, MD4, MD5, SHA-0 and SHA-1. In: De Prisco, R., Yung, M. (eds.) SCN 2006. LNCS, vol. 4116, pp. 242-256. Springer, Heidelberg (2006)

8. Rechberger, C., Rijmen, V.: On Authentication With HMAC and Non-Rondom Properties. In: Dietrich, S., Dhamija, R. (eds.) FC 2007 and USEC 2007. LNCS, vol. 4886, pp. 119-133. Springer, Heidelberg (2007)

9. Van Rompay, B., Biryukov, A., Preneel, B., Vandewalle, J.: Cryptanalysis of 3-pass HAVAL. In: Laih, C.-S. (ed.) ASIACRYPT 2003. LNCS, vol. 2894, pp. 228-245. Springer, Heidelberg (2003)

10. Wang, X., Feng, D., Lai, X., Yu, H.: Collisions for Hash Functions MD4, MD5, HAVAL-128 and RIPEMD, Cryptology ePrint Archive, Report 2004/199 (2007)

11. Wang, X., Feng, D., Yu, H.: The Collision Attack on Hash Function HAVAL-128. Science in China, Series E 35(4), 405-416 (2005)

12. Wang, X., Lai, X., Feng, D., Chen, H., Yu, X.: Cryptanalysis of the Hash Functions MD4 and RIPEMD. In: Cramer, R.J.F. (ed.) EUROCRYPT 2005. LNCS, vol. 3494, pp. 1-18. Springer, Heidelberg (2005)

13. Wang, X., Yin, X.Y., Yu, H.: Finding Collision in the Full SHA-1. In: Shoup, V. (ed.) CRYPTO 2005. LNCS, vol. 3621, pp. 17-36. Springer, Heidelberg (2005)

14. Wang, X., Yu, H., Yin, X.Y.: Efficient Collision Search Attacks on SHA-0. In: Shoup, V. (ed.) CRYPTO 2005. LNCS, vol. 3621, pp. 1-16. Springer, Heidelberg (2005)

15. Wang, X., Yu, H.: How to Break MD5 and Other Hash Functions. In: Cramer, R.J.F. (ed.) EUROCRYPT 2005. LNCS, vol. 3494, pp. 19-35. Springer, Heidelberg (2005)

16. Yu, H.: Cryptanalysis of Hash Functions and HMAC/NMAC, Doctoral dissertation, SHANDONG

17. Yu, H., Wang, X., Yun, A., Park, S.: Cryptanalysis of the Full HAVAL with 4 and 5 Passes. In: Robshaw, M.J.B. (ed.) FSE 2006. LNCS, vol. 4047, pp. 89-110. Springer, Heidelberg (2006)

18. Yu, H., Wang, G., Zhang, G., Wang, X.: The Second-Preimage Attack on MD4. In: Desmedt, Y.G., Wang, H., Mu, Y., Li, Y. (eds.) CANS 2005. LNCS, vol. 3810, pp. 1-12. Springer, Heidelberg (2005)

19. Zheng, Y., Pieprzyk, J., Seberry, J.: HAVAL - a one-way hashing algorithm with variable length of output. In: Zheng, Y., Seberry, J. (eds.) AUSCRYPT 1992. LNCS, vol. 718, pp. 83-104. Springer, Heidelberg (1993) 


\section{A Sufficient Conditions of the Second Preimage Differential Path of 3-Pass HAVAL}

Table 5 shows the sufficient conditions of the second preimage differential path of 3 -pass HAVAL, which are derived from the property of the Boolean function $f_{i}$ of appendix B. We solve and simplify the conditions of Table 5 and list the solutions in Table 6 .

\section{B Property of the Boolean Functions $f_{1}, f_{2}$ and $f_{3}$}

Recall that the input value of the $i$-th step is denoted $a_{i-8}, a_{i-7}, \cdots, a_{i-1}$ and the output value of the Boolean functions of the $i$-th step is denoted $t_{i}$. Tables 7 , 8 and 9 show the relations between the input difference and $t_{i}$ of the $i$-th step. In the column of Assumption in the Tables, $\mathbf{a}_{\mathbf{s}}[\mathbf{j}]$ represents the difference $\left(\Delta a_{i-8}, a_{i-7}, \cdots\right.$, $\left.\Delta a_{s}, \cdots, \Delta a_{i-1}\right)=\left(0,0, \cdots, a_{s}[j], 0, \cdots, 0\right)$ for $i-1 \leq s \leq i-7$ and $t_{i}[]$ means that the output difference of the Boolean function of the $i$-th step is zero (see Section 2.3 for the notations $a_{s}[j]$ and $\left.t_{i}[j]\right)$. Note that even though the sign is altered from $+j$ to $-j$ in both $\mathbf{a}_{\mathbf{s}}[\mathbf{j}]$ and $t_{i}[j]$, still the conditions are the same as in Tables 7. 8 and 9, however if the sign is altered only in one of $\mathbf{a}_{\mathbf{s}}[\mathbf{j}]$ and $t_{i}[j]$, the second conditions should be 1 (and the first ones are not altered). 
Table 5. Sufficient conditions of the second preimage differential path of 3-pass HAVAL

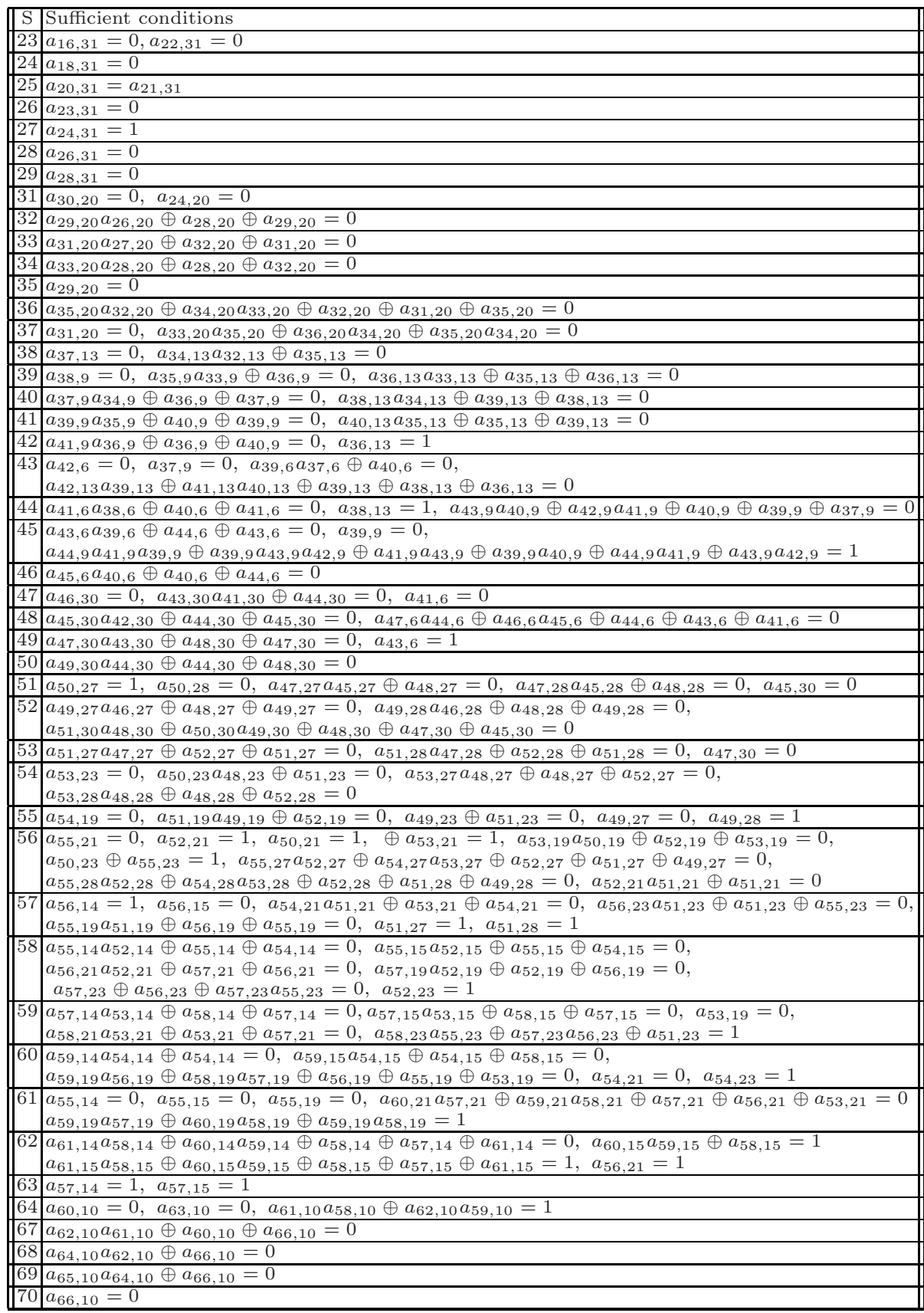


Table 6. Simplified sufficient conditions of the second preimage differential path of 3 pass HAVAL

\begin{tabular}{|c|c|}
\hline $\mathrm{S}$ S: & Sufficient conditions \\
\hline & $a_{16,31}=0, a_{18,31}=0, a_{20,31}=a_{21,31}, a_{22,31}=0$ \\
\hline 23 & $a_{23,31}=0$ \\
\hline 24 & $a_{24,31}=1, a_{24,20}=0$ \\
\hline 26 & $a_{26,31}=0$ \\
\hline 28 & $a_{28,31}=0, a_{28,20}=0$ \\
\hline 29 & $a_{29,20}=0$ \\
\hline 30 & $a_{30,20}=0$ \\
\hline 31 & $a_{31,20}=0$ \\
\hline 32 & $a_{32,20}=0$ \\
\hline 36 & $a_{36,13}=1, a_{36,9}=0, a_{34,20} a_{33,20} \oplus a_{35,20}=0$ \\
\hline 37 & $a_{37,9}=0, a_{37,6}=0, a_{37,13}=0, a_{33,20} a_{35,20} \oplus a_{36,20} a_{34,20} \oplus a_{35,20} a_{34,20}=0$ \\
\hline 38 & $a_{38,13}=1, a_{38,9}=0, a_{34,13} a_{32,13} \oplus a_{35,13}=0$, \\
\hline 39 & $a_{39,6}=1, a_{39,9}=0, a_{35,9} a_{33,9}=0, a_{33,13} \neq a_{35,13}$ \\
\hline 40 & $a_{40,9}=0, a_{40,6}=0, a_{34,13} \neq a_{39,13}$ \\
\hline 41 & $a_{41,6}=0, a_{40,13} a_{35,13} \oplus a_{35,13} \oplus a_{39,13}=0$ \\
\hline 42 & $a_{42,6}=0$ \\
\hline 43 & $a_{43,6}=1, a_{42,13} a_{39,13} \oplus a_{41,13} a_{40,13} \oplus a_{39,13}=0$ \\
\hline 44 & $a_{44,30}=0, a_{44,6}=0, a_{42,9} a_{41,9}=0$ \\
\hline 45 & $a_{45,6}=1, a_{45,30}=0, a_{41,9} a_{43,9} \oplus a_{44,9} a_{41,9} \oplus a_{43,9} a_{42,9}=1$ \\
\hline 46 & $a_{46,6}=1, a_{46,30}=0$ \\
\hline 47 & $a_{47,30}=0, a_{43,30} a_{41,30}=0$ \\
\hline 48 & $a_{48,30}=0$ \\
\hline 49 & $a_{49,27}=0, a_{49,28}=1$ \\
\hline 50 & $a_{50,21}=1, a_{50,27}=1, a_{50,28}=0$ \\
\hline 51 & $\begin{array}{l}a_{51,27}=1, a_{51,28}=1, a_{51,15}=0, a_{51,14}=0 \\
a_{47,27} a_{45,27} \oplus a_{48,27}=0, a_{47,28} a_{45,28} \oplus a_{48,28}=0\end{array}$ \\
\hline 52 & $a_{52,19}=0, a_{52,21}=1, a_{50,30} a_{49,30}=0, a_{52,23}=1, a_{48,27}=0, a_{46,28}=a_{48,28}$ \\
\hline 53 & $a_{53,19}=0, a_{53,14}=0, a_{53,15}=1, a_{53,21}=0, a_{53,23}=1, a_{47,28} \oplus a_{52,28}=1$ \\
\hline 54 & $\begin{array}{l}a_{54,14}=0, a_{54,15}=0, a_{54,19}=0, a_{50,23} a_{48,23} \oplus a_{51,23}=0, a_{52,27}=0 \\
a_{54,21}=0, a_{54,23}=1, a_{53,28} a_{48,28} \oplus a_{48,28} \oplus a_{52,28}=0\end{array}$ \\
\hline 55 & $a_{55,14}=0, a_{55,15}=0, a_{55,19}=0, a_{55,21}=0, a_{51,19} a_{49,19}=0, a_{49,23}=a_{51,23}$ \\
\hline 56 & $\begin{array}{l}a_{56,19}=0, a_{56,15}=0, a_{56,14}=1, a_{56,21}=1, a_{50,23}=a_{55,23} \\
a_{54,27} a_{53,27} \oplus a_{55,27}=1, a_{55,28} a_{52,28} \oplus a_{54,28} a_{53,28} \oplus a_{52,28} \oplus a_{51,28} \oplus a_{55,28}=0\end{array}$ \\
\hline 57 & $a_{57,14}=1, a_{57,15}=1, a_{57,21}=0, a_{56,23} a_{51,23} \oplus a_{51,23} \oplus a_{55,23}=0$ \\
\hline 58 & $a_{58,21}=1, a_{58,14}=0, a_{58,15}=0, a_{57,23} \oplus a_{56,23} \oplus a_{57,23} a_{55,23}=0$ \\
\hline 59 & $a_{59,21}=1, a_{58,23} a_{55,23} \oplus a_{57,23} a_{56,23} \oplus a_{55,23} \oplus a_{51,23}=1, a_{59,15}=1$ \\
\hline 60 & $a_{58,19} a_{57,19} \oplus a_{55,19}=0, a_{60,10}=0, a_{60,15}=1$ \\
\hline 61 & $a_{59,19} a_{57,19} \oplus a_{60,19} a_{58,19} \oplus a_{59,19} a_{58,19}=1, a_{61,15}=1$ \\
\hline 62 & $a_{60,14} a_{59,14} \oplus a_{61,14}=1$ \\
\hline 63 & $a_{63,10}=0$ \\
\hline 64 & $a_{61,10} a_{58,10} \oplus a_{62,10} a_{59,10}=1$ \\
\hline 66 & $a_{66,10}=0$ \\
\hline 67 & $a_{62,10} a_{61,10}=0$ \\
\hline 68 & $a_{64,10} a_{62,10}=0$ \\
\hline 69 & $a_{65,10} a_{64,10}=0$ \\
\hline
\end{tabular}


Table 7. Property of the Boolean function $f_{1}$

\begin{tabular}{|c|c|c|}
\hline \multicolumn{3}{|c|}{\begin{tabular}{l|l} 
ption & Conditions for satisfying the Assumption \\
\end{tabular}} \\
\hline \multirow[b]{2}{*}{$\mathbf{a}_{\mathbf{i}-1}[\mathbf{j}]$} & $t_{i}[$ & $a_{i-7}=0$ \\
\hline & $t_{i}[j]$ & $a_{i-7}=1, a_{i-3} a_{i-4} \oplus a_{i-2} a_{i-6} \oplus a_{i-5} a_{i-3} \oplus a_{i-5}=0$ \\
\hline \multirow[b]{2}{*}{$\mathbf{a}_{\mathbf{i}-2}[\mathbf{j}]$} & $t_{i}[$ & $a_{i-6}=0$ \\
\hline & $t_{i}[j]$ & $a_{i-6}=1, a_{i-3} a_{i-4} \oplus a_{i-7} a_{i-1} \oplus a_{i-5} a_{i-3} \oplus a_{i-5}=0$ \\
\hline \multirow[b]{2}{*}{$\mathbf{a}_{\mathbf{i}-3}[\mathbf{j}]$} & $t_{i}[$ & $a_{i-4}=a_{i-5}$ \\
\hline & $t_{i}[j]$ & $a_{i-4} \neq a_{i-5}, a_{i-1} a_{i-7} \oplus a_{i-6} a_{i-2} \oplus a_{i-5}=0$ \\
\hline \multirow[b]{2}{*}{$\mathbf{a}_{\mathbf{i}-4}[\mathbf{j}]$} & $t_{i}[$ & $a_{i-3}=0$ \\
\hline & $t_{i}[j]$ & $a_{i-3}=1, a_{i-1} a_{i-7} \oplus a_{i-6} a_{i-2} \oplus a_{i-5} a_{i-3} \oplus a_{i-5}=0$ \\
\hline \multirow[b]{2}{*}{$\mathbf{a}_{\mathbf{i}-5}[\mathbf{j}]$} & $t_{i}[$ & $a_{i-3}=1$ \\
\hline & $t_{i}[j]$ & $a_{i-3} a_{i-4} \oplus a_{i-3}=0, a_{i-1} a_{i-7} \oplus a_{i}$ \\
\hline \multirow[b]{2}{*}{$\mathbf{a}_{\mathbf{i}-6}[\mathbf{j}]$} & $t_{i}[]$ & $a_{i-2}=0$ \\
\hline & $t_{i}[j]$ & $a_{i-2}=1, a_{i-3} a_{i-4} \oplus a_{i-7} a_{i-1} \oplus a_{i-5} a_{i-3} \oplus a_{i-5}=0$ \\
\hline$[:]$ & $\overline{\mid t t_{i}[]}$ & $a_{i-1}=0$ \\
\hline
\end{tabular}

Table 8. Property of the Boolean function $f_{2}$

\begin{tabular}{|c|c|c|}
\hline issul & ion & Conditions for satisfying the Assumption \\
\hline & $t_{i}[]$ & $a_{i-4} a_{i-6} \oplus a_{i-3}=0$ \\
\hline$\| \mathbf{a}_{\mathbf{i}-1}[\mathbf{j}]$ & $t_{i}[j]$ & $a_{i-4} a_{i-6} \oplus a_{i-3}=1$ \\
\hline & & $a_{i-6} a_{i-2} a_{i-3} \oplus a_{i-4} a_{i-6} \oplus a_{i-4} a_{i-2}$ \\
\hline & & $\oplus a_{i-6} a_{i-5} \oplus a_{i-2} a_{i-3} \oplus a_{i-7} a_{i-6} \oplus a_{i-7}=0$ \\
\hline & $t_{i}[]$ & $a_{i-3} a_{i-6} \oplus a_{i-4} \oplus a_{i-3}=0$ \\
\hline$\| \mathbf{a}_{\mathbf{i}-2}[\mathbf{j}]$ & $t_{i}[j]$ & $a_{i-3} a_{i-6} \oplus a_{i-4} \oplus a_{i-3}=1$, \\
\hline & & $a_{i-4} a_{i-6} a_{i-1} \oplus a_{i-4} a_{i-6} \oplus a_{i-6} a_{i-5} \oplus a_{i-1} a_{i-3} \oplus a_{i-7} a_{i-6} \oplus a_{i-7}=0$ \\
\hline & $t_{i}[]$ & $\mid a_{i-2} a_{i-6} \oplus a_{i-1} \oplus a_{i-2}=0$ \\
\hline $\mid \mathbf{a}_{\mathbf{i}-3}[\mathbf{j}]$ & $t_{i}[j]$ & $a_{i-2} a_{i-6} \oplus a_{i-1} \oplus a_{i-2}=1$, \\
\hline & & $a_{i-4} a_{i-6} a_{i-1} \oplus a_{i-4} a_{i-6} \oplus a_{i-6} a_{i-5} \oplus a_{i-7} a_{i-6} \oplus a_{i-7}=0$ \\
\hline & $t_{i}[]$ & $a_{i-1} a_{i-6} \oplus a_{i-6} \oplus a_{i-2}=0$ \\
\hline$\| \mathbf{a}_{\mathbf{i}-4}[\mathbf{j}]$ & $t_{i}[j]$ & $a_{i-1} a_{i-6} \oplus a_{i-6} \oplus a_{i-2}=1$ \\
\hline & & $a_{i-6} a_{i-2} a_{i-3} \oplus a_{i-6} a_{i-5} \oplus a_{i-1} a_{i-3} \oplus a_{i-2} a_{i-3} \oplus a_{i-7} a_{i-6} \oplus a_{i-7}=0$ \\
\hline & $t_{i}[]$ & $a_{i-6}=0$ \\
\hline$\| a_{i-5}[\mathbf{j}]$ & $t_{i}[j]$ & $a_{i-6}=1$, \\
\hline & & $a_{i-4} a_{i-6} a_{i-1} \oplus a_{i-6} a_{i-2} a_{i-3} \oplus a_{i-4} a_{i-6}$ \\
\hline & & $\oplus a_{i-4} a_{i-2} \oplus a_{i-1} a_{i-3} \oplus a_{i-2} a_{i-3} \oplus a_{i-7} a_{i-6} \oplus a_{i-7}=0$ \\
\hline & $t_{i}[]$ & $a_{i-1} a_{i-4} \oplus a_{i-2} a_{i-3} \oplus a_{i-4} \oplus a_{i-5} \oplus a_{i-7}=0$ \\
\hline $\mid \mathbf{a}_{\mathbf{i}-6}[\mathbf{j}]$ & $t_{i}[j]$ & $a_{i-1} a_{i-4} \oplus a_{i-2} a_{i-3} \oplus a_{i-4} \oplus a_{i-5} \oplus a_{i-7}=1$, \\
\hline & & $a_{i-4} a_{i-2} \oplus a_{i-1} a_{i-3} \oplus a_{i-2} a_{i-3} \oplus a_{i-7}=0$ \\
\hline & $t_{i}[]$ & $a_{i-6}=1$ \\
\hline $\mid \mathbf{a}_{\mathbf{i}-7}[\mathbf{j}]$ & $t_{i}[j]$ & $a_{i-6}=0$ \\
\hline & & $\begin{array}{l}a_{i-4} a_{i-5} a_{i} \oplus a_{i-6} a_{i-2} a_{i-3} \oplus a_{i-4} a_{i-6} \\
\quad \oplus a_{i-4} a_{i-2} \oplus a_{i-6} a_{i-5} \oplus a_{i-1} a_{i-3} \oplus a_{i-2} a_{i-3}=0\end{array}$ \\
\hline
\end{tabular}


Table 9. The property of the Boolean function $f_{3}$

\begin{tabular}{|c|c|c|}
\hline & & fions for satisfyin \\
\hline & $t_{i}[]$ & $a_{i-4}=1$ \\
\hline $\mathrm{a}_{\mathbf{i}-1}[\mathrm{j}]$ & $t_{i}[j]$ & $a_{i-4}=0, a_{i-4} a_{i-5} a_{i-6} \oplus a_{i-6} a_{i-3} \oplus a_{i-5} a_{i-2} \oplus a_{i-4} a_{i-7}=0$ \\
\hline & $t_{i}[]$ & $a_{i-5}=0$ \\
\hline$a_{i-2}[j]$ & $t_{i}[j]$ & $a_{i-5}=1, a_{i-6} a_{i-3} \oplus a_{i-4} a_{i-7} \oplus a_{i-1} a_{i-4} \oplus a_{i-1}=0$ \\
\hline & $t_{i}[]$ & $a_{i-6}=0$ \\
\hline $\mathrm{a}_{\mathrm{i}-3}[\mathrm{j}]$ & $t_{i}[j]$ & $a_{i-6}=1, a_{i-4} a_{i-5} a_{i-6} \oplus a_{i-5} a_{i-2} \oplus a_{i-4} a_{i-7} \oplus a_{i-1} a_{i-4} \oplus a_{i-1}=0$ \\
\hline & $t_{i}[]$ & $a_{i-5} a_{i-6} \oplus a_{i-7} \oplus a_{i-1}=0$ \\
\hline $\mathrm{a}_{\mathrm{i}-4}[\mathrm{j}]$ & $t_{i}[j]$ & $a_{i-5} a_{i-6} \oplus a_{i-7} \oplus a_{i-1}=1, a_{i-6} a_{i-3} \oplus a_{i-5} a_{i-2} \oplus a_{i-1}=0$ \\
\hline & $t_{i}[]$ & $a_{i-4} a_{i-6} \oplus a_{i-2}=0$ \\
\hline$a_{i-5}[j]$ & $t_{i}[j]$ & $a_{i-4} a_{i-6} \oplus a_{i-2}=1, a_{i-6} a_{i-3} \oplus a_{i-4} a_{i-7} \oplus a_{i-1} a_{i-4} \oplus a_{i-1}=0$ \\
\hline & $t_{i}[]$ & $a_{i-4} a_{i-5} \oplus a_{i-3}=0$ \\
\hline$a_{i-6}[j]$ & $t_{i}[j]$ & $a_{i-4} a_{i-5} \oplus a_{i-3}=1, a_{i-5} a_{i-2} \oplus a_{i-4} a_{i-7} \oplus a_{i-1} a_{i-4} \oplus a_{i-1}=0$ \\
\hline & $t_{i}[]$ & $a_{i-4}=0$ \\
\hline$l_{i-7}[\mathbf{j}]$ & $t_{i}[j]$ & $a_{i-4}=1, a_{i-4} a_{i-5} a_{i-6} \oplus a_{i-6} a_{i-3} \oplus a_{i-5} a_{i-2} \oplus a_{i-1} a_{i-4} \oplus a_{i-1}=0$ \\
\hline
\end{tabular}

